\title{
Application of the Euler Lagrange Formalism for Determining the Equation of Motion in the Case of Radial Fall into a Non-Rotating, Charged Black Hole
}

\author{
A. Sfarti \\ CS Dept., 387 Soda Hall, UC Berkeley, USA \\ Email: egas@pacbell.net
}

\begin{abstract}
In this paper we set to accomplish two things: determine the equation of motion for an uncharged test probe falling radially into a charged, non-rotating black hole and determine the relationship between coordinate acceleration and coordinate speed. The paper is concerned only what happens outside the event horizon, since we are using only the external Reissner-Nordstrom equations in the derivations. What happens inside the event horizon (the presence of a wormhole connecting the black hole to a white hole) is outside the scope of this paper.
\end{abstract}

Keywords: General relativity, Reissner-Nordstrom metric, Euler-Lagrange formalism.

PACS: $03.30 .+\mathrm{p}, 52.20 . \mathrm{Dq}, 52.70 . \mathrm{Nc}$.

\section{Introduction}

We will present a method based on the Lagrangian for the derivation of the equation of motion of an uncharged test probe falling radially into a charged, non-rotating black hole. In a prior paper [1] we have derived the solution for the case of non-rotating, non-charged black holes. In the following, we are extending the derivation to the case of charged black holes. While we could have started from the geodesic equation, the derivation based on the Euler-Lagrange equations is more intuitive and less prone to error. The Reissner-Nordstrom metric for the particular case of absence of rotation $(d \theta=d \varphi=0)$ is $[2,6-8]$ :

$$
\begin{aligned}
& d s^{2}=\alpha d t^{2}-\frac{1}{\alpha} d r^{2} \\
& \alpha=1-\frac{r}{r}+\frac{r_{Q}^{2}}{r^{2}}
\end{aligned}
$$

where $r_{s}=\frac{2 G M}{c^{2}}$ is the Schwarzschild radius, $r_{Q}^{2}=\frac{G Q^{2}}{4 \pi \varepsilon_{0} c^{4}}$ where $\mathrm{G}$ is the universal attraction constant, $\mathrm{Q}$ is the black hole charge, $\mathrm{r}$ is the radial coordinate, $\mathrm{c}$ is the speed of light in vacuum and $\boldsymbol{\varepsilon}_{0}$ is the vacuum electric permittivity. From the metric we obtain, as shown in $[1,2]$ :

a) the Lagrangian

$$
L=\alpha{\frac{d t^{2}}{d s^{2}}}^{2}-\frac{1}{\alpha} \frac{d r^{2}}{d s^{2}}
$$

The Lagrangian (2) is obtained following an idea by Rindler [3], whereby one replaces the parameter $t$ with the arc length $s$ along the solution curve, provided that that curve isn't null. This allows replacement of the Lagrangian $L=\sqrt{g_{i j} \dot{x}_{i} \dot{x}_{j}}$ with its square $L=g_{i j} \dot{x}_{i} \dot{x}_{j}$ where the overdot represents now derivative with respect to the arc length $s$. Rindler proves that the Euler-Lagrange equations for $L=\sqrt{g_{i j} \dot{x}_{i} \dot{x}_{j}}$ are equivalent to those for $L=g_{i j} \dot{x_{i}} \dot{x}_{j}[3]$. 
b) from the Lagrangian we obtain the Euler-Lagrange system of equations [1,2]:

and, respectively:

$$
\begin{aligned}
& \frac{d}{d s}\left(\frac{\partial L}{\partial \dot{t}}\right)-\frac{\partial L}{\partial t}=0 \\
& \frac{d}{d s}\left(\frac{\partial L}{\partial \dot{r}}\right)-\frac{\partial L}{\partial r}=0
\end{aligned}
$$

$$
\begin{aligned}
& \frac{d}{d s}\left(\alpha \frac{d t}{d s}\right)=0 \\
& \alpha \frac{d t}{d s}=k \\
& \frac{d}{d s}\left(\frac{\partial L}{\partial \dot{r}}\right)-\frac{\partial L}{\partial r}=\frac{d}{d s}\left(\frac{-2 \dot{r}}{\alpha}\right)-t^{2} \frac{d \alpha}{d r}+\dot{r}^{2} \frac{d}{d r}\left(\frac{1}{\alpha}\right)= \\
& =2\left(-\frac{\ddot{r}}{\alpha}+\dot{r^{2}} \frac{\frac{d \alpha}{d r}}{\alpha^{2}}\right)-\dot{r}^{2} \frac{\frac{d \alpha}{d r}}{\alpha^{2}}-t^{2} \frac{d \alpha}{d r}=-2 \frac{\ddot{r}}{\alpha}+\dot{r}^{2} \frac{\frac{d \alpha}{d r}}{\alpha^{2}}-\dot{t^{2}} \frac{d \alpha}{d r}
\end{aligned}
$$

The over-dots signify derivative with respect to $s$. From the metric (1.1) we obtain:

$$
\alpha\left(\frac{d t}{d s}\right)^{2}=1+\frac{1}{\alpha}\left(\frac{d r}{d s}\right)^{2}
$$

Substituting (1.5) into (1.4) we obtain

c) the equation of motion:

From (1.1) we obtain:

$$
\frac{d^{2} r}{d s^{2}}+\frac{1}{2} \frac{d \alpha}{d r}=0
$$

$$
\begin{aligned}
& \left(\frac{d r}{d s}\right)^{2}=\alpha^{2}\left(\frac{d t}{d s}\right)^{2}-\alpha \\
& \frac{d r}{d s}=\sqrt{k^{2}-\alpha}
\end{aligned}
$$

In $(1.7) \mathrm{k}$ can be determined by setting the condition that the coordinate (or proper) speed is zero when the particle is dropped from radial distance $r_{0}$ towards the mass $\mathrm{M}$ :

Therefore proper speed is:

$$
\begin{aligned}
& 0=\frac{d r}{d s}=\sqrt{k^{2}-\alpha\left(r_{0}\right)} \\
& k=\sqrt{\alpha\left(r_{0}\right)}
\end{aligned}
$$

Finally, the equation of motion is:

$$
\frac{d r}{d s}=\sqrt{\alpha\left(r_{0}\right)-\alpha(r)}=\sqrt{\frac{r_{s}}{r}-\frac{r_{Q}^{2}}{r^{2}}-\frac{r_{s}}{r_{0}}+\frac{r_{Q}^{2}}{r_{0}^{2}}}
$$

$$
\frac{d^{2} r}{d s^{2}}=\frac{-\frac{d \alpha}{d s}}{2 \sqrt{k^{2}-\alpha}}=-\frac{1}{2 \sqrt{k^{2}-\alpha}} \frac{d \alpha}{d r} \frac{d r}{d s}=-\frac{1}{2}\left(\frac{r_{s}}{r^{2}}-\frac{2 r_{Q}^{2}}{r^{3}}\right)
$$

Equation (1.10) is the equation of motion expressed in terms of the proper acceleration $\frac{d^{2} r}{d s^{2}}$. A quick comparison with the results $[1,2,5]$ for non-charged, non-rotating black holes shows that the right hand 
side of the equation of motion changes from $-\frac{1}{2} \frac{r_{s}}{r^{2}}$ to $-\frac{1}{2}\left(\frac{r_{s}}{r^{2}}-\frac{2 r_{Q}^{2}}{r^{3}}\right)$. This change is perfectly intuitive if we consider that in both cases, the equation of motion [1,2,5], expressed in terms of $\alpha$ is given by (1.6). It is interesting to note that the charge of the black hole contributes an acceleration that is inversely proportional to the cube of the radial distance and it is of opposite sense to the standard gravitational acceleration. This contribution exists even though the test probe is uncharged.

\section{Discussion}

Although charged black holes with $r_{Q} \ll r_{\mathrm{S}}$ are similar to the Schwarzschild black hole, it is known that they have two horizons: the event horizon and an internal Cauchy horizon. As with the Schwarzschild metric, the event horizons for the spacetime are located where the metric component $\alpha$ diverges; that is, where:

$$
0=\alpha=1-\frac{r_{s}}{r}+\frac{r_{Q}^{2}}{r^{2}}
$$

The above equation has two solutions, each corresponding to one horizon:

$$
r=0.5\left(r_{s} \pm \sqrt{r_{s}^{2}-4 r_{Q}^{2}}\right)
$$

Black holes with $2 r_{Q}>r_{\mathrm{S}}$ are believed not to exist in nature because they would contain a naked singularity, in our paper we will consider only the physically realistic case $2 r_{Q} \leq r_{s}<r$ which corresponds to:

$$
0<\alpha<1
$$

In other words, our paper deals only with the realistic case of radial fall outside the external event horizon of a physically realizable charged hole, one that would not contain a naked singularity.

\section{$3 \quad$ The Dependency between Coordinate Acceleration and Coordinate Speed in Reissner-Nordstrom Coordinates}

In this section we determine the relationship between coordinate acceleration and coordinate speed. Using (1.1) and (1.3) the coordinate speed is:

$$
\begin{aligned}
& \left(\frac{d r}{d t}\right)^{2}=\alpha^{2}-\alpha\left(\frac{d s}{d t}\right)^{2} \\
& \frac{d r}{d t}=\sqrt{\alpha^{2}-\alpha\left(\frac{d s}{d t}\right)^{2}}=\sqrt{\alpha^{2}-\frac{\alpha^{3}}{k^{2}}}=\alpha(r) \sqrt{1-\frac{\alpha(r)}{\alpha\left(r_{0}\right)}}
\end{aligned}
$$

From (2.1) we get the coordinate acceleration:

$$
\begin{aligned}
& \frac{d^{2} r}{d t^{2}}=\frac{d}{d s}\left(\frac{d r}{d t}\right) \frac{d s}{d t}=\frac{2 \alpha-\frac{3 \alpha^{2}}{k^{2}}}{2 \sqrt{\alpha^{2}-\frac{\alpha^{3}}{k^{2}}}} \frac{d \alpha}{d s} \frac{\alpha}{k}=\frac{\alpha}{k} \frac{2 \alpha-\frac{3 \alpha^{2}}{k^{2}}}{2 \frac{\alpha}{k} \sqrt{k^{2}-\alpha}}\left(\frac{r_{s}}{r^{2}}-\frac{2 r_{Q}^{2}}{r^{3}}\right) \frac{d r}{d s}= \\
& =\frac{2 \alpha-\frac{3 \alpha^{2}}{k^{2}}}{2}\left(\frac{r_{s}}{r^{2}}-\frac{2 r_{Q}^{2}}{r^{3}}\right)
\end{aligned}
$$

Eliminating $k$ between (3.1) and (3.2) we obtain:

$$
\frac{d^{2} r}{d t^{2}}=\frac{1}{2}\left(\frac{3}{\alpha}\left(\frac{d r}{d t}\right)^{2}-\alpha\right)\left(\frac{r_{s}}{r^{2}}-\frac{2 r_{Q}^{2}}{r^{3}}\right)
$$


So, the relationship between coordinate acceleration and coordinate speed in the case of radial motion into a charged, non-rotating black hole is:

$$
\frac{d^{2} r}{d t^{2}}-\frac{3\left(\frac{r_{s}}{r^{2}}-\frac{2 r_{Q}^{2}}{r^{3}}\right)}{2\left(1-\frac{r_{s}}{r}+\frac{r_{Q}^{2}}{r^{2}}\right)}\left(\frac{d r}{d t}\right)^{2}+\frac{1}{2}\left(1-\frac{r_{s}}{r}+\frac{r_{Q}^{2}}{r^{2}}\right)\left(\frac{r_{s}}{r^{2}}-\frac{2 r_{Q}^{2}}{r^{3}}\right)=0
$$

A quick sanity check shows that for zero charge $\left(r_{Q}=0\right)$ we recover the equation for the Schwarzschild case developed in [1]. An alternative expression for the coordinate acceleration as a function of the radial coordinate can be obtained from (3.1) and (3.3):

$$
\frac{d^{2} r}{d t^{2}}=\alpha(r)\left(1-\frac{3 \alpha(r)}{2 \alpha\left(r_{0}\right)}\right)\left(\frac{r_{s}}{r^{2}}-\frac{2 r_{Q}^{2}}{r^{3}}\right)
$$

As per (2.3):

$$
\begin{aligned}
& 2 r_{Q} \leq r_{s}<r \\
& 0<\alpha<1
\end{aligned}
$$

Another useful formula can be derived from (3.1):

$$
\frac{d t}{d r}=\frac{1}{\alpha(r) \sqrt{1-\frac{\alpha(r)}{\alpha\left(r_{0}\right)}}}
$$

Formula (3.7) proves essential in determining the radial separation for two test probes falling into a black hole [5]. Elementary algebra shows that under conditions (3.6) the expression:

$$
1-\frac{\alpha(r)}{\alpha\left(r_{0}\right)}>0
$$

is always positive, so the equation (3.7) has physically realizable solutions.

\section{Conclusion}

We have provided a derivation of the relationship between coordinate acceleration and coordinate speed for an uncharged test probe falling into a charged, non-rotating black hole. We have also shown that the charge of the black hole contributes an acceleration that is inversely proportional to the cube of the radial distance and it is counter the sense to the standard gravitational acceleration. This contribution exists even though the test probe is uncharged. We have used the same formalism in order to derive the equation of motion of the test probe.

\section{References}

1. A. Sfarti, "Application of the Euler-Lagrange method in determination of the coordinate acceleration", EJP, 37, 3, 2016

2. A. Sfarti, "Euler-Lagrange Solution for Calculating Particle Orbits in Gravitational Fields", Fizika A, 19, 4, 2010

3. Rindler,W. "Relativity-Special,General and Cosmological", 2006, Oxford Press, pp205

4. R.A.Mould, "Basic Relativity", Springer-Verlag, 2002, p329.

5. A Sfarti, "The Two Test Probe Chase". JAPSI, 6, 4, 2016

6. D. Raine, E. Thomas, "Black Holes", Imperial College Press, 2005

7. J.B. Hartle, "Gravity: An Introduction to Einstein's General Relativity", 2003, Addison-Wesley

8. S Chandrashekhar, The Mathematical Theory of Black Holes, Oxford University Press Inc., 2000 